\title{
Tunneling spectroscopy on the correlation effects in FeSi
}

\author{
M. Fäth, J. Aarts, A. A. Menovsky, ${ }^{*}$ G. J. Nieuwenhuys, and J. A. Mydosh \\ Kamerlingh Onnes Laboratory, Leiden University, P.O. Box 9504, 2300 RA Leiden, The Netherlands
}

(Received 22 August 1997)

\begin{abstract}
We have performed electron tunneling spectroscopy on FeSi single crystals in the temperature range 4-300 $\mathrm{K}$ by using a scanning tunneling microscope. The differential conductance $(d I / d V)$, when corrected for Schottky barrier effects, exhibits two strongly temperature-dependent peaks on either side of the Fermi level that emerge below $\approx 200 \mathrm{~K}$ and that are separated by a (pseudo)gap of $\approx 50 \mathrm{meV}$. Our observations can be ascribed to the formation of quasiparticle density of states caused by $d$-electron correlation. The tunneling spectra are in good agreement with photoemission spectroscopy as both techniques probe the correlated $d$-electron density of states (DOS). Our results are also consistent with optical reflectivity data and Raman spectroscopy, which, in contrast, are sensitive to the conduction (c) electron DOS. [S0163-1829(98)08248-4]
\end{abstract}

\section{INTRODUCTION}

For more than half a century the cubic transition-metal compound FeSi has provoked continuous interest due to its unusual and still unexplained magnetic and electronic properties. ${ }^{1}$ One of the most striking features is its magnetic susceptibility, which rises nearly exponentially up to a temperature of approximately $500 \mathrm{~K}$, where it exhibits a broad maximum, and for still higher temperatures, decreases obeying a Curie-Weiss law. ${ }^{2}$ The intrinsic magnetic susceptibility, from which a paramagnetic Curie-tail has been subtracted, ${ }^{3}$ vanishes below $50 \mathrm{~K}$, and the possibility of a transition to antiferromagnetic order at high temperatures has been ruled out by neutron diffraction, ${ }^{4}$ the ${ }^{57} \mathrm{Fe}$ Mössbauer effect,,${ }^{3,5}{ }^{27} \mathrm{Si}$ NMR, ${ }^{3,6}$ and $\mu^{+}$SR (Ref. 7) measurements. Therefore, alternative reasons for this nonmagnetic behavior must be sought in the formation of a spin gap or in the disappearance of local moments due to, e.g., spin fluctuations or a high/low spin transition. The electrical resistivity at room temperature is high $(\approx 200 \mu \Omega \mathrm{cm})$, as for a dirty metal, and rises several orders of magnitude upon cooling, indicating a narrow gap semiconductor. Even though conventional band-structure calculations $^{8,9}$ yield an indirect semiconducting gap that could be responsible for the thermally activated behavior of the resistivity at low temperatures, they are not able to account for other experimental observations.

Several theoretical models have been suggested to explain the unusual temperature dependence of the susceptibility. These include the many-body theory of thermally induced spin fluctuations ${ }^{10-12}$ where magnetic moments become thermally activated and eventually saturate at high temperatures, approaching local moment behavior with a Curie-Weiss susceptibility. Neutron scattering experiments ${ }^{13,14}$ have confirmed the concept of temperature-induced magnetism, and the low-temperature noninteracting quasiparticles. However, this theory as well as more phenomenological single-electron approaches $^{2,15}$ need to assume a priori the existence of two narrow bands at the gap edges of a broad semiconductorlike background density of states (DOS) in order to properly describe magnetic and transport properties. Such a model DOS is justified in a Kondo insulator description ${ }^{16,17}$ where the hybridization of the conduction band with an $f$ level leads to a strong renormalization of the bands at the edges of the hybridization gap around the Fermi energy. Due to the striking similarities in the physical properties between FeSi and so-called "Kondo insulators,", ${ }^{8,19}$ it is tempting to place $\mathrm{FeSi}$ in the latter class of materials, originally comprising only rare-earth compounds, with the Fe $3 d$ electrons taking over the role of the hybridized rare-earth $4 f$ electrons.

Evidence for the importance of correlation effects in $\mathrm{FeSi}$ at low temperatures first came from optical reflectivity measurements. ${ }^{20}$ Those indicated the existence of a charge gap in FeSi that, however, is mostly filled in at a lower temperature than is expected from thermal activation processes in an ordinary semiconductor. Also, the electronic spectral weight removed by the gap is redistributed over an energy range much larger than the gap size.

In this work we present for the first time the results of electron tunneling experiments on FeSi. Tunneling spectroscopy is able to probe charge excitations and their temperature dependence. Therefore, we compare our results with recent photoemission experiments ${ }^{21}$, optical reflectivity, ${ }^{20,22}$ and Raman-scattering data. ${ }^{23}$ Our interpretation of the results of these different spectroscopic techniques will be based on a model for spectroscopies of correlated electron systems, recently presented by Rozenberg, Kotliar, and Kajueter. ${ }^{17}$ In this model, FeSi is treated in the framework of the periodic Anderson lattice, which leads to a distinction between the response of the conduction $(c)$ electrons which form a wide band, and the more localized and correlated $d$ electrons. The $d$-electron partial DOS shows the opening of a gap, accompanied by the formation of strong and narrow quasiparticle bands. A gap also evolves in the (thermodynamic) $c$-electron partial DOS upon lowering the temperature, which is probed especially by optical reflectivity and which also strongly affects the transport and thermodynamic properties. We argue that single-particle tunneling is particularly sensitive to the correlated $d$-electron states, and that our foremost observation is the formation of these narrow bands. This makes tunneling spectroscopy a powerful technique, complementary to optical spectroscopy. 

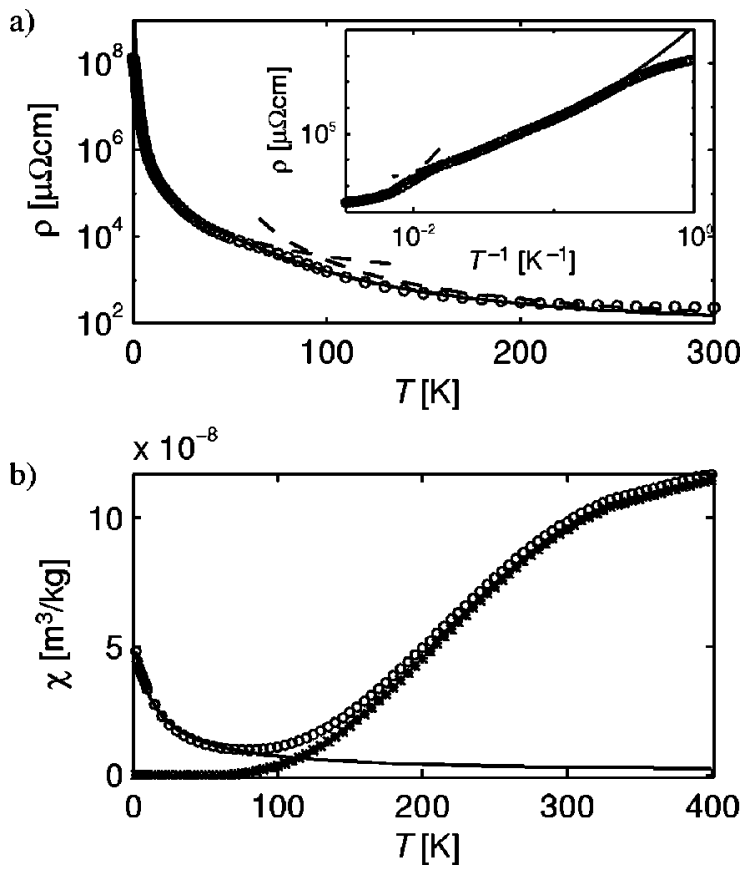

FIG. 1. (a) Electrical resistivity vs temperature of an FeSi single crystal on which tunneling and optical reflectivity measurements were performed. The solid line represents a fit to the data points (circles) using a combined model of variable range hopping and thermal activation (dashed lines) as described in the text. Inset: semilogarithmic plot of the resistivity vs inverse temperature. (b) Magnetic dc susceptibility vs temperature in a field of $0.1 \mathrm{~T}$. The experimental data (circles) at low temperatures have been fitted with a Curie-Weiss law (solid line). The corrected data (stars) after subtraction of the Curie-Weiss tail represent the intrinsic susceptibility of $\mathrm{FeSi}$.

\section{EXPERIMENTS}

\section{A. Sample preparation and characterization}

We have investigated single crystals grown from the stoichiometric melt either by the floating zone method using a light image furnace in a purified argon atmosphere or by a modified tri-arc Czochralski method. ${ }^{24}$ The cubic crystal symmetry $^{2}$ of $\mathrm{FeSi}$, a distorted rocksalt structure, was confirmed by $\mathrm{x}$-ray diffraction. Electron-probe microanalysis showed a stoichiometric and homogeneous single phase.

The electrical resistivity [Fig. 1(a)] increases by typically five orders of magnitude on cooling from room temperature to $0.1 \mathrm{~K}$. The absolute value of the resistivity saturates below $1 \mathrm{~K}$ and is sample dependent due to minor imperfections of the crystal. ${ }^{6}$ The temperature dependence, and especially the weak shoulder around $60 \mathrm{~K}$, cannot be described by a single mechanism. The best fit is obtained by using an expression for variable range hopping $\left\{\rho(T) \propto \exp \left[\left(T_{0} / T\right)^{1 / 4}\right]\right\}$ for the low-temperature regime and semiconductorlike thermal activation $\left[\rho(T) \propto \exp \left(\Delta_{R} / 2 k_{\mathrm{B}} T\right)\right]$ at higher temperatures. Fitting the data with a temperature-independent gap yields values of $\Delta_{R}=830 \mathrm{~K}$ and $T_{0} \approx 10^{5} \mathrm{~K}$. We should stress here that this description in terms of variable range hopping and thermal activation is purely phenomenological, intended to parametrize $\rho(T)$ for comparison with other data. For instance, the value for $T_{0}$ is sample dependent and probably a measure for the residual disorder; its high value would lead to a very small localization length, but the variable range hopping model cannot be applied rigorously because of the electron correlation effects to be discussed below. Also at the high temperatures there are several problems. The fit is not very satisfactory around and above room temperature, because the resistivity actually starts to increase again with increasing temperature; ${ }^{25}$ the value of $\Delta_{R}$ is much too large to be explained by nearest-neighbor hopping in a localized semiconductor; nor can it be simply taken as a measure for the semiconducting gap: both the optical reflectivity data and the tunneling measurements (see below) show that the use of a temperature-independent gap does not capture the essential physics of FeSi.

The magnetic susceptibility [Fig. 1(b)] shows the typical and gradual falloff below $400 \mathrm{~K}$ along with a rise at low temperatures, which obeys the Curie-Weiss law $\chi(T)$ $=\chi_{0}+C /(T-\Theta)$ and is attributed to paramagnetic impurities. ${ }^{3}$ It became clear from NMR (Ref. 6) and $\mu^{+}$SR (Ref. 7) measurements that the magnetic susceptibility at low temperatures has a different origin than that at high temperatures. This is because plots of $\mu^{+}$SR Knight shift versus susceptibility reveal a different slope for both temperature regions while the line shape of the ${ }^{29} \mathrm{Si}$ spin-echo spectrum changes from asymmetric to symmetric on going from low to high temperatures. In our case the low-temperature susceptibility could be attributed to approximately 0.3 at. \% moments of $3 \mu_{B}$ with a paramagnetic Curie temperature of about $\Theta=-14 \mathrm{~K}$. The magnetization versus field at $2 \mathrm{~K}$ exhibits a narrow hysteresis loop in external fields below $0.1 \mathrm{~T}$. The maximum magnetization of this irreversible part could be accounted for by only $20 \mathrm{ppm}$ of free Fe ferromagnetic moments with $3 \mu_{B}$. However, the magnetization does not saturate at higher fields (not shown) but increases linearly up to $5 \mathrm{~T}$, corresponding to a susceptibility of $2.8 \times 10^{-8} \mathrm{~m}^{3} / \mathrm{kg}$. This indicates spin-fluctuating moments instead of paramagnetic ones. After subtraction of the Curie-Weiss tail the remaining magnetic susceptibility, which is believed to be intrinsic, ${ }^{3}$ vanishes at low temperatures. Thermally activated susceptibility begins below room temperature and its $T$ curvature smoothens to approach the expected peak around $500 \mathrm{~K}^{2}{ }^{2}$ The high resistivity ratio $\rho(T \rightarrow 0) / \rho(300 \mathrm{~K}) \approx 2$ $\times 10^{5}$ together with the small amount of magnetic impurities indicates good sample quality.

\section{B. Tunneling spectroscopic measurements}

The tunneling measurements were carried out by means of a scanning tunneling microscope (STM) that allowed the adjustment of both the tunneling resistance (typically $1 \mathrm{nA}$ current at $0.4 \mathrm{~V}$ bias) and the position on the sample surface where the $I-V$ spectra are recorded. We will discuss the probable type of contact below. An electrochemically etched tip from gold, silver, or platinum wire served as a counterelectrode to which the bias voltage was applied. Immediately prior to mounting the sample, the single crystals were cleaved in order to obtain a clean surface with arbitrary crystallographic orientation. In most cases, in situ cleaved samples showed smoother surfaces than samples that were mechanically polished. By changing the positions of the tip over the surface, even by several micrometers at low temperatures, it was proven that the $I-V$ spectra are independent of the actual location, thereby demonstrating the homogeneity of the tunneling barrier. This represents an improvement 


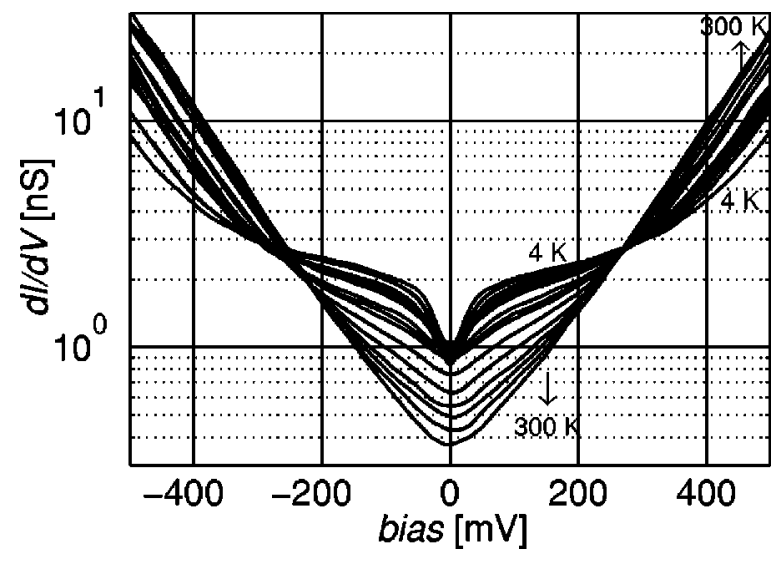

FIG. 2. Differential conductance curves of an FeSi-Au tunnel junction at different temperatures between $4 \mathrm{~K}$ and $300 \mathrm{~K}$. (Corresponding temperatures of the curves from top to bottom at high bias: $298 \mathrm{~K}, 287 \mathrm{~K}, 234 \mathrm{~K}, 204 \mathrm{~K}, 198 \mathrm{~K}, 191 \mathrm{~K}, 176 \mathrm{~K}, 172 \mathrm{~K}$, $164 \mathrm{~K}, 146 \mathrm{~K}, 134 \mathrm{~K}, 122 \mathrm{~K}, 105 \mathrm{~K}, 59 \mathrm{~K}, 4 \mathrm{~K}$.)

compared to preliminary STM attempts ${ }^{26}$ on FeSi, where the tunneling curves did depend on the surface position. There the surface was cleaned by $\mathrm{Ar}^{+}$ion etching but not broken in situ.

Figure 2 shows differential conductance spectra at various temperatures between $4 \mathrm{~K}$ and room temperature. The derivative $d I / d V$ was numerically calculated from the measured voltage-current curves. A positive bias voltage means electrons tunneling preferably from $\mathrm{FeSi}$ into the tip. The rapid exponential increase of the conductance with bias voltage is different from what is usually found in metalinsulator-metal (MIM) junctions (with the insulator either an insulating material or a vacuum gap), where the behavior at low bias is Ohmic. Rather, it is reminiscent of tunneling through a metal-semiconductor junction and the formation of a Schottky barrier. ${ }^{27}$ In such a junction, charge is transferred from the semiconductor to the metallic tip, leaving a depletion region in which the electrostatic potential varies quadratically with distance from the interface. For a degenerate (highly doped) semiconductor the Schottky barrier acts as tunneling barrier. Since the thickness of the depletion layer decreases with increasing applied voltage, the voltage dependence of the barrier transmission is not negligible, leading to strong non-Ohmic behavior at relatively low bias.

The theory for the $I-V$ characteristics of Schottky barriers is well developed. ${ }^{27-29}$ In the case of $\mathrm{FeSi}$, we find that the spectra near room temperature can be fully described by treating the material as a heavily $n$-doped semiconductor, with very reasonable numbers for the different parameters involved. Furthermore, we find that the contribution of thermionic emission over the barrier can be neglected in comparison to the contribution from the tunneling current, because of the thin depletion region. This explains why the $I-V$ characteristics are nearly symmetric instead of exhibiting rectification. The junction type appears to be (vacuum) tunneling rather than point contact although the actual tipsample separation is probably very small due to the presence of the Schottky barrier. We infer this mainly from the two observations that the tunneling current is exponentially dependent on the tip-sample separation and that topographic scanning is possible without noticeable dragging of, or damage to, the tip.
One possible concern in measuring such high resistivity samples is that the bias voltage is not only applied over the tunneling barrier, but also over part of the sample, leading to distorted $I-V$ characteristics. This can occur especially when the current distribution in the area immediately below the tip is inhomogeneous, e.g., due to the presence of (nonconducting) grain boundaries, microcracks, or strongly layered structures (high- $T_{c}$ materials are a good example of the latter). Signatures of such problems are irreproducible results at different positions on the surface or on different samples. Our data proved highly reproducible, probably because the samples were single crystals with a homogeneous and isotropic structure. Furthermore we did not observe any changes in the differential conductance for different tip-to-sample distances, corresponding to tunneling resistances between $300 \mathrm{M} \Omega$ and $1.2 \mathrm{G} \Omega$ normalized at $0.4 \mathrm{~V}$ bias. This makes us confident that the resistance of the tunneling barrier was much larger than the series resistance of the sample volume below the tip. For a more detailed description of the data analysis in the presence of a Schottky barrier we refer to the Appendix. Also discussed there is the temperature dependence of the differential conductance. In essence, lowering the temperature lowers the conductance, but the slope in the semilogarithmic plot of conductance versus bias voltage remains constant, as seen in Fig. 2 above about $400 \mathrm{mV}$. The Schottky barrier model, therefore, explains the conductance at high bias, for all temperatures, in terms of the bias dependence of the barrier transmission factor. This in turn allows us to eliminate the effect of the barrier on the measured curves at all voltages by following an approach similar to the one first suggested by Stroscio and co-workers for spectroscopy on semiconductor surfaces. ${ }^{30,31}$ An approximate expression for the tunneling current from a small tip is given by ${ }^{32}$

$$
I=C \int_{0}^{e V} \rho(E) D(E) d E,
$$

with $C$ a constant, $\rho(E)$ the local density of states, and $D(E)$ the transmission probability. The differential conductance then is

$$
\frac{d I}{d V}=C \rho(e V) D(e V),
$$

showing that density-of-states features can be found from dividing the differential conductance by the transmission probability.

In our case, $D(E)$ is given by the Schottky barrier and is fully known at room temperature. At lower temperatures, we can fit the Schottky barrier model to the high bias part (above $400 \mathrm{mV}$ ) of the measured curve and again obtain $D(E)$ (see Appendix for details). Dividing the measured $d I / d V$ by $D(E)$ then yields the density of states connected with the tunneling electrons. More accurately, it yields the change in this density of states with respect to room temperature. The results are shown in Fig. 3.

Around $300 \mathrm{~K}$, the renormalized spectra are almost featureless, and display good agreement with the Schottky barrier fit at all bias voltages. The salient feature of our tunneling spectra is their strong temperature dependence below about $200 \mathrm{~K}$. This is illustrated by the appearance of two distinct and narrow conductance peaks on either side of the 


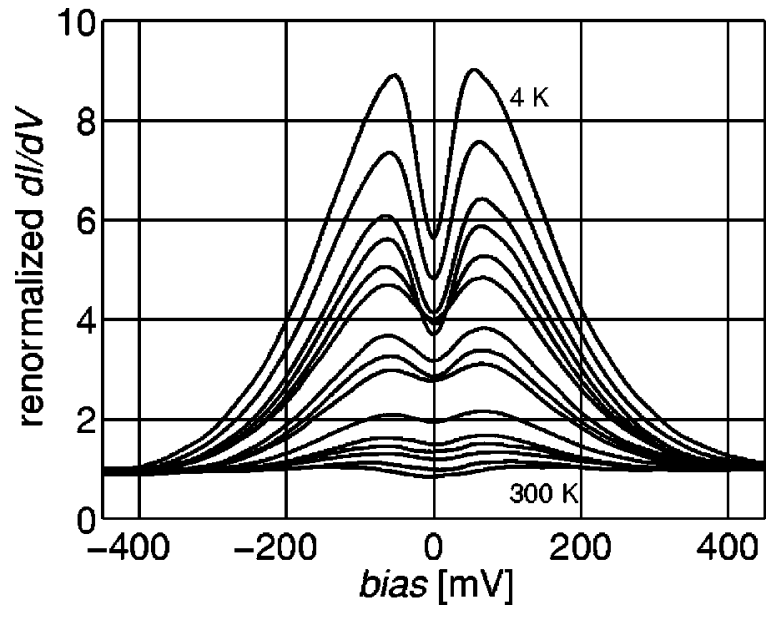

FIG. 3. Temperature dependence of the quasiparticle density of states after renormalizing the differential conductance spectra in Fig. 2 by the appropriate Schottky barrier transmission factor (see text). The spectra were taken at temperatures of $298 \mathrm{~K}, 287 \mathrm{~K}$, $234 \mathrm{~K}, 204 \mathrm{~K}, 198 \mathrm{~K}, 191 \mathrm{~K}, 176 \mathrm{~K}, 172 \mathrm{~K}, 164 \mathrm{~K}, 146 \mathrm{~K}, 134 \mathrm{~K}$, $122 \mathrm{~K}, 105 \mathrm{~K}, 59 \mathrm{~K}$, and $4 \mathrm{~K}$.

Fermi level where a well-defined minimum forms, see Fig. 3. The dip at zero bias becomes increasingly more pronounced on lowering the temperature, but the conductance remains finite. However, the tunneling data do not allow us to distinguish, whether the dip is a gap or a pseudogap in the density of states, since imperfections in the barrier (leakage current) and noise-induced current might also contribute to a finite slope of the conductance at zero bias. Moreover, our normalization method used to handle the exponential change of the (Schottky) barrier tunneling conductance tends to overestimate the zero bias conductance at low temperatures. This we will discuss in the Appendix. The main point is that the absolute value of the renormalized zero bias conductance should not be considered as precise. If we view the raw data in Fig. 2 we see that above $\approx 140 \mathrm{~K}$ the zero bias conductance increases on lowering the temperature whereas below $\approx 140 \mathrm{~K}$ it decreases. We will return to this issue later on. Meanwhile we refer to the dip as a gap. The main point to be discussed next is the origin of the formation of the conductance peaks and the gap in between. In order to accomplish this, we will first compare the tunneling spectra of Fig. 3 to those obtained by other spectroscopic techniques.

\section{Comparison to other spectroscopic techniques}

Photoemission experiments ${ }^{21,33}$ revealed not only an energy gap, but also a strongly temperature-dependent peak near the band edge of the valence band in $\mathrm{FeSi}$, which resembles our tunneling conductance peak at low (plus) bias. From an analysis of the spectral line shape, Saitoh et al. $^{33}$ concluded that a large mass renormalization and the appearance of a sharp peak in the quasiparticle DOS occur near the valence-band edge at low temperatures, probably due to strong electron correlation. They found that the photoemission spectra are dominated by the Fe $3 d$ partial DOS. This leads us to conjecture that it is the interacting $d$-electron quasiparticle DOS which is responsible for the narrow peaks around the Fermi energy at low temperatures seen in both photoemission and tunneling spectroscopy. The large angular dependence of the emission peak observed by Park et al. ${ }^{21}$ with angle-resolved photoemission on single-crystalline samples is not likely to be found by tunneling, because the directionality of the tunneling process is mostly lost if one of the electrodes is a semiconductor (or a semimetal) as is the case for FeSi, certainly at low temperatures. ${ }^{34}$ Thus, tunneling probes the quasiparticle DOS of $\mathrm{FeSi}$, but integrated over a larger angle than can be obtained with photoemission. This partly explains our observation of a much broader peak in comparison to the very small dispersion found by photoemission at optimum analyzer angle. Additionally, the quasiparticle lifetime could be reduced due to scattering, e.g., at the interfacial barrier, which would also lead to a broader peak. In our tip-sample configuration the valence-band side of $\mathrm{FeSi}$ is mapped onto positive bias voltages, but our peaks, corresponding to both valence and conduction bands, are approximately symmetric with respect to the Fermi level.

This then suggests that the quasiparticle DOS is symmetric with respect to the Fermi energy. In that case, the size of the gap inferred from the photoemission spectra is ca. $50 \mathrm{meV}$. From the tunneling spectra, we obtain an upper limit of $110 \mathrm{meV}$, by using the voltage difference between the peak positions. Taking into account that the peaks in the quasiparticle DOS will not coincide with the gap edges and rather using the inflection points in the conductance rise, we find a gap width of about $50 \mathrm{meV}$. We take this as good overall agreement between the two types of measurements. Very recent photoemission measurements at room temperature ${ }^{35}$ revealed the existence of both a flat and a dispersive band near the Fermi energy, which could provide a conduction band crossing a localized electron level. This is prerequisite for the formation of a hybridization gap in a Kondo insulator.

Optical reflectivity experiments ${ }^{20,36,22}$ performed on single crystals were the first direct measurements which revealed the opening of a charge gap in FeSi. Schlesinger et $a ._{.}{ }^{20}$ and Damascelli et $a l^{22}$ essentially agree in their main observation, to wit, the gap disappears at a much lower temperature $(\approx 200 \mathrm{~K})$ than would be expected for a conventional semiconductor with a gap size of $60 \mathrm{meV}$ (Ref. 20) to $70 \mathrm{meV}$ (Ref. 22) at low temperatures. Another unusual feature that also suggests the presence of strong correlations in $\mathrm{FeSi}$ is that the spectral weight expelled from the gap region is spread over a frequency range more than $1 \mathrm{eV}$ above the gap. The results by DeGiorgi et al. ${ }^{36}$ are in disagreement with those findings, but as was noted earlier their sample is apparently more metallic and probably not representative for the intrinsic behavior of $\mathrm{FeSi}^{19}{ }^{19} \mathrm{We}$, therefore, compare our results below to those obtained by Damascelli et al. ${ }^{22}$ who performed their measurements on the same high-quality single crystals that we used for tunneling spectroscopy.

In order to give an approximate description of the gap evolution with temperature, we adopt the integrated area under the quasiparticle DOS peaks (see Fig. 3) as the distinguishing parameter. With respect to other parameters, e.g., peak heights, the area data show less scattering throughout the numerous spectra taken on different samples and with different tip materials. Figure 4 shows the integrated peak area as a function of temperature. All data points follow one averaged curve, which evidences that the tunneling measurements are not significantly influenced by tip or sample dependent properties in contrast to resistivity or susceptibility measurements. As can clearly be seen, the correlation effects mainly develop below $\approx 200 \mathrm{~K}$ and have approached their 


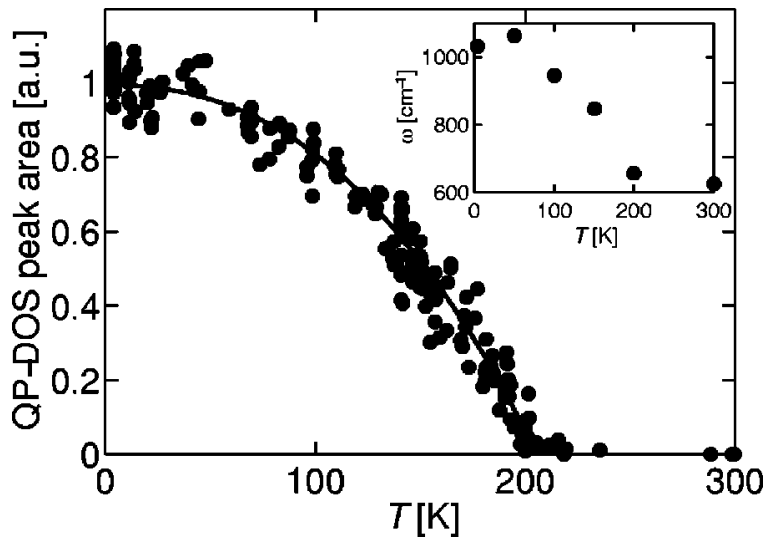

FIG. 4. Temperature dependence of the quasiparticle DOS area under the renormalized conduction curves (see Fig. 3) for various tunnel junctions between different $\mathrm{FeSi}$ single crystals and metallic tips. The solid line serves as a guide to the eye. Inset: position of the center of the optical conductivity shoulder obtained from optical reflectivity measurements (Ref. 22) performed on one of the same samples that were used for tunneling measurements.

maximum amplitude below $\approx 50 \mathrm{~K}$. Plotted in the inset of Fig. 4 is the position of the center of the optical conductivity shoulder versus temperature obtained from optical reflectivity measurements of Damascelli et al. ${ }^{22}$ Although this "gap" parameter is arbitrary, its temperature dependence compares well with the quasiparticle DOS area. It is obvious that the development of the direct charge gap (optical conductivity) and the appearance of the quasiparticle DOS (photoemission and tunneling spectroscopy) have the same onset temperature. This indicates that both effects are related to each other and have a common origin.

A gap in the charge excitation spectrum has also been found by electronic Raman scattering. ${ }^{23}$ While its variation with temperature is in good agreement with the above results, its size $(\approx 94 \mathrm{meV})$ is estimated to be somewhat larger. We note that the observed substantial increase of spectral weight above the gap could also be attributed to quasiparticle excitations. The energy range of $0.3 \mathrm{eV}$ beyond the gap, which is covered by the excess of spectral weight at low temperatures, and the position of the maxima in the Ramanscattering intensity near $130 \mathrm{meV}$ both are comparable to our tunneling spectra with respect to their energy and temperature dependence. However, the electronic Raman scattering differs from the optical conductivity where the spectral weight is not piled up in a maximum just beyond the gap, but is spread out over a much larger range of energy.

\section{DISCUSSION}

The connection between the peaks in the photoemission and tunneling spectra, on the one hand, and the gap measured by optical reflectivity and Raman scattering, on the other, may be illuminated by a recent theory of Rozenberg, Kotliar, and Kajueter ${ }^{17}$ based on the periodic Anderson model in a local impurity self-consistent approximation. According to this theory, which describes a possible scenario for the physics of $\mathrm{FeSi}$, the direct band gap $\Delta_{\text {dir }}$ experienced by the conduction $(c)$ electrons is merely a small dip without peaks in the $c$-partial DOS, and can be detected by optical conductivity. $\Delta_{\text {dir }}$ is still a pseudogap at elevated temperatures with a finite but low DOS at the Fermi level, which is indicated by both the Schottky barrier effect in the tunneling characteristics and the negative slope $d \rho / d T$ of the resistivity up to room temperature. As this gap in the $c$-partial DOS develops upon cooling below $200 \mathrm{~K}$, there is a more dramatic effect whereby narrow bands of quasiparticles are formed due to the interactions, i.e., strong correlations, between the $d$ electrons and this causes a major change in the $d$-partial DOS. Two large peaks emerge at low temperatures above and below $E_{F}$ with a well-defined, steep minimum in between. Thus, an indirect (pseudo)gap $\Delta_{\text {ind }}$ appears in the much larger and peaked $d$-partial DOS. Within this theoretical framework we relate the $\Delta_{\text {ind }}$ to the photoemission and tunneling spectra while only the $\Delta_{\text {dir }}$ of the $c$-partial DOS can be observed by the optical conduction since it is a process without change of momentum. Note that angular resolved photoemission and tunneling spectroscopy are momentum transferring processes and can detect $\Delta_{\text {ind }}$, if $\Delta_{\text {ind }}$ $<\Delta_{\text {dir }}$. From the theory of Rozenberg, Kotliar, and Kajueter ${ }^{17} \Delta_{\text {ind }}$ is expected to be less than $\Delta_{\text {dir }}$.

Therefore, our tunneling measurements are directly probing the electronic excitation spectrum of the highly correlated $d$ electrons. This appears valid not only for the peaked structure at low temperatures, but also for the growth of the low-energy $d$-partial DOS at intermediate temperatures. Between $200 \mathrm{~K}$ and $140 \mathrm{~K}$, the buildup of states at low bias occurs faster than the development of the gap, in complete agreement with theoretical calculations. ${ }^{17}$

From the same calculations, it is seen that the quasiparticle ( $d$-partial) DOS is a few times larger than the conduction electron (c-partial) DOS and thus changes in the $d$-partial DOS can be expected to dominate in the tunneling characteristics. More significant, however, is the fact that the $d$-partial DOS is the result of many-body correlation effects (due to local Coulomb repulsion) while the conduction band has substantially uncorrelated single-particle character. In the latter case, the tunneling current is only weakly sensitive to density-of-states effects, since a higher (lower) DOS is cancelled by a lower (higher) particle velocity, as pointed out by Harrison $^{34}$ and Wolf. ${ }^{27}$ Therefore, the tunneling characteristics reflects primarily the quasiparticle DOS. The same conclusion was recently drawn from measurements ${ }^{37}$ on $\mathrm{La}_{0.7} \mathrm{Ca}_{0.3} \mathrm{MnO}_{3}$ where substantial correlations in the $\mathrm{Mn} d$ band exist.

We stress that the increase of DOS at low temperatures is only in the correlated $d$ part, and that this is not in contradiction to the approximately activated behavior of the bulk electrical conductivity (resistivity). The latter involves the thermodynamic DOS and will be very sensitive to the gap in the $c$-partial DOS. Tunneling (or photoemission) as a singleparticle process, has a quite different relation to the thermodynamic DOS, especially in strongly correlated materials.

Finally we address the apparent increase of spectral weight upon formation of the peaked quasiparticle DOS at low temperatures. It is likely that the DOS is taken from high energies that lie outside the experimentally accessible energy window of our tunneling measurements in analogy to the missing spectral weight in optical reflectivity measurements. ${ }^{20}$ In the periodic Anderson model, the full energy width over which the $d$-partial DOS is found to change is set by the $c$-band width and the interaction ener- 


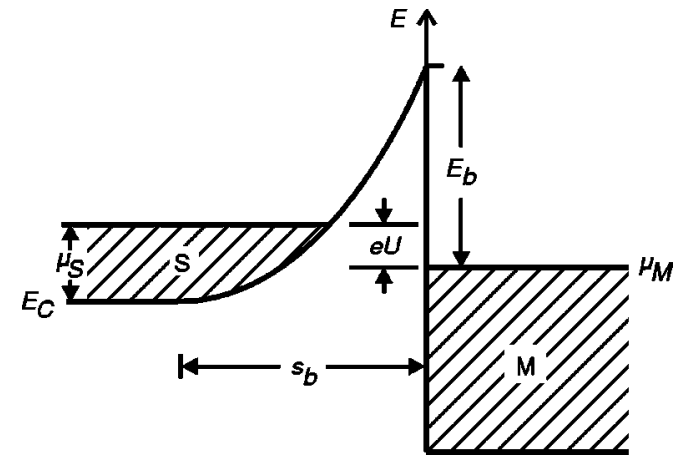

FIG. 5. Metal (M)-semiconductor (S) contact with a bias voltage $(U)$ applied in the forward direction. $E_{C}$ represents the conduction-band edge of the semiconductor with its chemical potential $\mu_{S}$ and depletion layer width $s_{b}$. The barrier height $E_{b}$ is measured from the chemical potential $\mu_{M}$ of the metal.

gies, both of the order of eV's and much larger than our bias voltage range.

\section{SUMMARY}

In summary, we have investigated FeSi single crystals by electron tunneling spectroscopy. The appearance of two distinct peaks in the tunneling conductance mainly below $200 \mathrm{~K}$, which are separated by an indirect gap of $\approx 50 \mathrm{meV}$, could be ascribed to the formation of a quasiparticle DOS of interacting $d$ electrons in agreement with photoemission spectroscopy. In contrast to tunneling and photoemission, optical reflectivity measurements do not probe the quasiparticle excitations but detect the direct band gap seen by conduction electrons. Its onset temperature and evolution with decreasing temperature is very similar to that of the quasiparticle DOS. This suggests that both gaps, direct and indirect, have the same origin, while the indirect gap appears to be slightly smaller than the direct gap. None of the spectroscopic results is in agreement with a conventional semiconductor description. Instead the underlying physics of $\mathrm{FeSi}$ seems to be described within a Kondo insulator model where strong electron correlations are taken into account.

\section{ACKNOWLEDGMENTS}

We thank A. Damascelli, D. van der Marel, and J. Zaanen for stimulating discussions and B. Becker for resistance measurements below $1 \mathrm{~K}$.

\section{APPENDIX: TUNNELING THROUGH A SCHOTTKY BARRIER}

Here we present some details of the analysis of the data in Fig. 2 in terms of tunneling through a Schottky barrier. Especially, we will discuss the strongly nonlinear conductance arising from such a barrier, and show that a simple model can fully describe the experiments at high temperature.

The basic band picture of a metal-semiconductor contact is sketched in Fig. 5. Band bending occurs in the semiconductor near the interface, driven by the difference in work function between the two materials and the adjustment of the chemical potentials. The bending leads to a transfer of charge to the metal, leaving, in an $n$-type semiconductor, a posi-

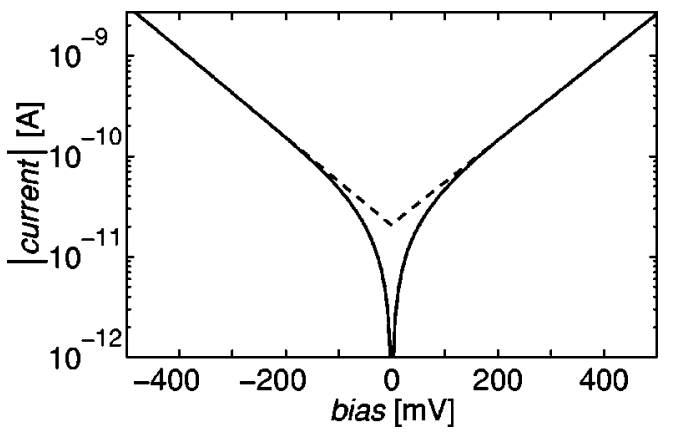

FIG. 6. Tunneling spectrum taken at room temperature (solid curve) with a linear fit (dashed line) to the logarithm of the high bias part above approximately $150 \mathrm{meV}$.

tively charged space region. Sufficiently high doping of the semiconductor (also appropriate for the case of $\mathrm{FeSi}$ ) places it on the metallic side of the Mott transition and shifts the chemical potential above the lower conduction-band edge. The ensuing tunneling barrier has a transmission factor that differs from MIM barriers since not only the mean height but also the width of the barrier depends on the bias voltage. The tunneling problem for a parabolic shape on the semiconductor side was solved exactly by Conley et al. ${ }^{38}$ In this model, the barrier width $s_{b}(U)$ is given by

$$
s_{b}=\sqrt{\frac{2 \epsilon\left(E_{b}+\mu_{s}-U\right)}{e^{2} N_{d}}},
$$

with $\epsilon$ the static dielectric constant of the material, $N_{d}$ the donor density, $E_{b}$ the barrier height as measured from the metal chemical potential $\left(\mu_{M}\right), \mu_{S}$ the semiconductor chemical potential, and $U$ the bias voltage, defined in such a way that forward biasing lowers the barrier seen from the semiconductor; this corresponds to positive (tip) bias in our measurements. As we will see below, the appropriate value for $E_{b}+\mu_{S}$ to describe the measurements is about $5.3 \mathrm{eV}$; together with values of $\epsilon \approx 200 \epsilon_{0}$ from optical measurements $^{22}$ and $N_{d} \approx 10^{28} / \mathrm{m}^{3}$ from Hall effect measurements, ${ }^{39}$ we find a barrier width $s_{b}(0)$ of about 3 $\mathrm{nm}$. This is a high but thin barrier, and it ensures that tunneling is the important contribution to the current, rather than thermionic emission over the top of the barrier. For this case, simplified expressions for the transmission factor and the tunneling current were given by Padovani and Stratton. ${ }^{40,29} \mathrm{It}$ turns out that (in the tunneling case), the current can be expressed as

$$
I=C_{0} I_{s}(U, T) e^{e|U| / E_{00}},
$$

with $C_{0}$ a constant, $I_{s}(U, T)$ a very weakly voltage- and temperature-dependent prefactor, and $E_{00}$ an energy characteristic for the semiconductor, given by

$$
E_{00}=\frac{e \hbar}{2} \sqrt{\frac{N_{d}}{\epsilon m^{*}}} .
$$

Here, $m^{*}$ is the effective mass of the tunneling electron; all other symbols have their usual meaning.

In order to compare this with the measurements, Fig. 6 shows the spectrum taken at $300 \mathrm{~K}$ in a semilog plot, along 
with a linear fit to the high bias regime, corresponding to neglecting the bias voltage dependence of $I_{s}$. Clearly, deviations only occur below about $150 \mathrm{mV}$; above that, we find $E_{00} \approx 0.1 \mathrm{eV}$. Substituting this value in Eq. (A3), with $\epsilon$ and $N_{d}$ as given above, we find $m^{*} \approx 1.7 m_{e}$, indicating that the tunneling electrons in this regime are mainly uncorrelated conduction band electrons. Note that a value of $0.1 \mathrm{eV}$ for $E_{00}$ is significantly higher than usually found for semiconductors, which is essentially due to the small depletion region.

Taking now the full expression for $I_{s}(U, T)$ from Ref. 29, we can fit the measurement over the whole voltage range, as shown in Fig. 7 for the current [Fig. 7(a)] and the differential conductance [Fig. 7(b)]. The additional parameters in the fit are $E_{b} \approx 5 \mathrm{eV}$ and $\mu_{S} \approx 0.3 \mathrm{eV}$. The value for the barrier height is quite large, which is probably due to the small contact or a small additional vacuum gap. The model could be applied to the data without the need to incorporate an additional resistance due to a vacuum gap in series with the Schottky barrier. This means that in spite of the scanning possibility, which excludes a mechanical contact, the actual tip-sample distance is very small. With these same parameters and by only varying $T$, we can calculate the spectra for $170 \mathrm{~K}$ and $4 \mathrm{~K}$. The results are also shown in Fig. 7. The effect of decreasing the temperature is to shift the differential conductance downwards, while the slope in the semilog plot remains constant. Qualitatively, the experimental spectra at high bias show the same behavior. Quantitatively, the high bias conductance can be reproduced down to about $150 \mathrm{~K}$. Below this temperature the measured values become lower than the calculated ones, with a difference of maximally $30 \%$ at $4 \mathrm{~K}$. Also, the onset of deviation from linearity shifts to higher bias, emphasizing the growing importance of the density-of-states effects. For the calculation of the transmission factor in Eq. (2) we used $C_{0}$ in Eq. (A2) as a fit parameter rather than as a constant (fixed at $300 \mathrm{~K}$ ) in order to shift the calculated spectrum onto the measured one at high bias.

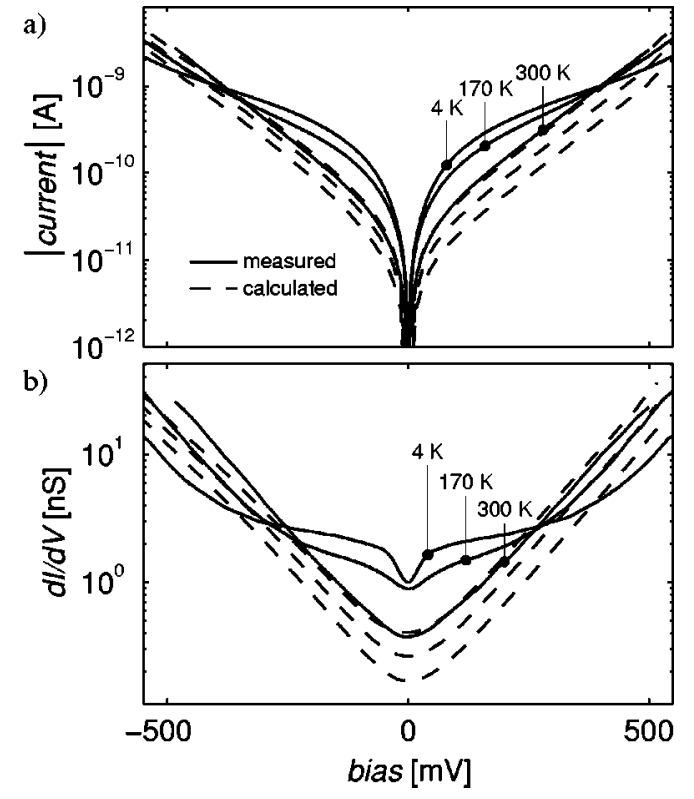

FIG. 7. Experimental tunneling spectra (solid curves) and calculated spectra (dashed curves) using the Schottky barrier model for (a) current vs bias voltage and (b) differential conductance vs bias voltage. The temperature of the experiments is indicated in the plots. The same temperature was used for the calculations: $300 \mathrm{~K}$ (top), $170 \mathrm{~K}$ (middle), $4 \mathrm{~K}$ (bottom).

However, this implies an even larger inaccuracy when calculating the renormalized conductance near zero bias since the measured data are divided by very small numbers. Our fits based on the above theoretical expressions ${ }^{29}$ are most imprecise at very low energies and generally tend to underestimate the measured value. Therefore, around zero bias and inside the gap the renormalized values are too high. In contrast, the peaks are nicely represented since they are located at energies where the Schottky barrier model yields a good description of the transmission probability.
*Also at Van der Waals-Zeeman Laboratory, University of Amsterdam, Valckenierstraat 67, 1018 XE Amsterdam, The Netherlands.

${ }^{1}$ M. B. Hunt, M. A. Chernikov, E. Felder, H. R. Ott, Z. Fisk, and P. Canfield, Phys. Rev. B 50, 14933 (1994).

${ }^{2}$ V. Jaccarino, G. K. Wertheim, J. H. Wernick, L. R. Walker, and S. Arajs, Phys. Rev. 160, 476 (1967).

${ }^{3}$ G. K. Wertheim, V. Jaccarino, J. H. Wernick, J. A. Seitchik, H. J. Williams, and R. C. Sherwood, Phys. Lett. 18, 89 (1965).

${ }^{4}$ H. Watanabe, H. Yamamoto, and K. Ito, J. Phys. Soc. Jpn. 18, 995 (1963).

${ }^{5}$ I. Vincze (private communication).

${ }^{6}$ S. Takagi, H. Yasuoka, S. Ogawa, and J. H. Wernick, J. Phys. Soc. Jpn. 50, 2539 (1981).

${ }^{7}$ G. J. Nieuwenhuys et al. (unpublished).

${ }^{8}$ L. F. Mattheiss and D. R. Hamann, Phys. Rev. B 47, 13114 (1993).

${ }^{9}$ C. Fu, M. P. C. M. Krijn, and S. Doniach, Phys. Rev. B 49, 2219 (1994).

${ }^{10}$ Y. Takahashi and T. Moriya, J. Phys. Soc. Jpn. 46, 1451 (1979).

${ }^{11}$ S.N. Evangelou and D. M. Edwards, J. Phys. C 16, 2121 (1983).
${ }^{12}$ Y. Takahashi, J. Phys.: Condens. Matter 9, 2593 (1997).

${ }^{13}$ G. Shirane, J. E. Fischer, Y. Endoh, and K. Tajima, Phys. Rev. Lett. 59, 351 (1987).

${ }^{14}$ K. Tajima, Y. Endoh, J. E. Fischer, and G. Shirane, Phys. Rev. B 38, 6954 (1988)

${ }^{15}$ D. Mandrus, J. L. Sarrao, a. Migliori, J. D. Thompson, and Z. Fisk, Phys. Rev. B 51, 4763 (1995).

${ }^{16}$ Y. Ōno, T. Matsuura, and Y. Kuroda, J. Phys. Soc. Jpn. 63, 1406 (1994).

${ }^{17}$ M. J. Rozenberg, G. Kotliar, and H. Kajueter, Phys. Rev. B 54, 8452 (1996).

${ }^{18}$ G. Aeppli and Z. Fisk, Comments Condens. Matter Phys. 16, 155 (1992).

${ }^{19}$ Z. Fisk, J. L. Sarrao, S. L. Cooper, P. Nyhus, G. S. Boebinger, A. Passner, and P. C. Canfield, Physica B 223\&224, 409 (1996).

${ }^{20}$ Z. Schlesinger, Z. Fisk, H. T. Zhang, M. B. Maple, J. F. DiTusa, and G. Aeppli, Phys. Rev. Lett. 71, 1748 (1993).

${ }^{21}$ C. H. Park, Z. X. Shen, A. G. Loeser, D. S. Dessau, D. G. Mandrus, A. Migliori, J. Sarrao, and Z. Fisk, Phys. Rev. B 52, 16981 (1995).

${ }^{22}$ A. Damascelli, K. Schulte, D. van der Marel, and A. A. Men- 
ovsky, Phys. Rev. B 55, R4863 (1997).

${ }^{23}$ P. Nyhus, S. L. Cooper, and Z. Fisk, Phys. Rev. B 51, 15626 (1995).

${ }^{24}$ A. A. Menovsky and J. J. M. Franse, J. Cryst. Growth 65, 286 (1983)

${ }^{25}$ B. C. Sales, E. C. Jones, B. C. Chakoumakos, J. A. FernandezBaca, H. E. Harmon, and J. W. Sharp, Phys. Rev. B 50, 8207 (1994).

${ }^{26}$ J. Aarts and A. P. Volodin, Physica B 206\&207, 43 (1995).

${ }^{27}$ E. L. Wolf, Principles of Electron Tunneling Spectroscopy (Oxford University Press, Oxford, 1985).

${ }^{28}$ C. B. Duke, in Tunneling in Solids, Vol. 10 of Solid State Physics: Advances in Research and Applications, edited by F. Seitz, D. Turnbull, and H. Ehrenreich (Academic Press, New York, 1969).

${ }^{29}$ F. A. Padovani, in Semiconductors and Semimetals, edited by R. Willardson and A. Beer (Academic, New York, 1971), Vol. 7, pp. 75-146.

${ }^{30}$ J. A. Stroscio, R. M. Feenstra, and A. P. Fein, Phys. Rev. Lett. 57, 2579 (1986).

${ }^{31}$ J. A. Stroscio and R. M. Feenstra, in Scanning Tunneling Micros- copy, Vol. 27 of Methods of Experimental Physics, edited by J. A. Stroscio and W. J. Kaiser (Academic Press, San Diego, 1993).

${ }^{32}$ A. Selloni, P. Carnevali, E. Tosatti, and C. D. Chen, Phys. Rev. B 31, 2602 (1985).

${ }^{33}$ T. Saitoh, A. Sekiyama, T. Mizokawa, A. Fujimori, K. Ito, H. Nakamura, and M. Shiga, Solid State Commun. 95, 307 (1995).

${ }^{34}$ W. A. Harrison, Phys. Rev. 123, 85 (1961).

${ }^{35}$ G. R. Castro, J. Alvarez, M. E. Davilá, M. C. Asensio, and E. G. Michel, J. Phys.: Condens. Matter 9, 1871 (1997).

${ }^{36}$ L. DeGiorgi, M. B. Hunt, H. R. Ott, M. Dressel, B. J. Feenstra, G. Grüner, Z. Fisk, and P. Canfield, Europhys. Lett. 28, 341 (1994).

${ }^{37}$ J. Y. T. Wei, N.-C. Yeh, and R. P. Vasquez, Phys. Rev. Lett. 79, 5150 (1997).

${ }^{38}$ J. W. Conley, C. B. Duke, G. D. Mahan, and J. J. Tiemann, Phys. Rev. 150, 466 (1966).

${ }^{39}$ S. Paschen, E. Felder, M. A. Chernikov, H. R. Ott, Z. Fisk, and J. Sarrao, Czech. J. Phys. 46, 1997 (1996).

${ }^{40}$ F. A. Padovani and R. Stratton, Solid-State Electron. 9, 695 (1966). 